\title{
USING MD CAD OBJECTS TO AUTOMATICALLY GENERATE A CONTRACT-DRIVEN SCHEDULE
}

\author{
Chung-Wei Feng, Associate Professor \\ Dept. of Civil Engineering \\ National Cheng Kung University \\ No.1 Ta-Hsueh Rd. Tainan 70101, Taiwan. \\ cfeng@mail.ncku.edu.tw
}

\author{
Yi-Jao Chen, Ph.D Candidate \\ Dept. of Civil Engineering \\ National Cheng Kung University \\ No.1 Ta-Hsueh Rd. Tainan 70101, Taiwan. \\ yjchen@mail.leader.edu.tw
}

\begin{abstract}
To efficiently and effectively manage projects in the construction phase, project managers have to transfer information within the drawings to obtain a project schedule. However, practically, the result is usually arbitrary depending on the project scheduler's experiences. This research employs MD CAD objects as basic scheduling components to develop a model that can effectively generate a contract-driven schedule. Several principles are developed to obtain Object Relationship Matrix (ORM) and Object Similarity Matrix (OSM). Genetic algorithms are then used with ORM and OSM to generate the optimal sequence of constructing building components. Several constructive constraints, such as the resource limitation and the continuity of workflow, are also taken into consideration within the model development. And the Project Planning and Scheduling System (PPSS) is developed to enhance the practice of the proposed model. In addition, a case study is presented to examine the efficiency of PPSS. It is proved that the automated scheduling model is capable to generate an optimal contract-driven schedule, and provides multi-dimensional information for management.
\end{abstract}

Keywords: MD Model, Construction Schedule, Genetic Algorithm (GA)

\section{INTRODUCTION}

In the pre-construction phase, preparing a workable and detailed construction schedule is crucial to the success of the construction project. However, practically, most construction schedules are unable to fulfill the actual needs in terms of the construction management. One of the reasons is the difficulty of effectively and efficiently transferring the information within the drawings to obtain a suitable project schedule for construction. Transferring the information within the drawings to obtain the construction schedule usually requires different participants repeatedly perform the integration procedure according to the actual construction condition (Dawood et al. 2002). As a result, retrieving information, such as quantities of different resources, and the method to perform construction, within the drawings is not an easy task and usually time consuming. Comparing with the quantity takeoff, the factors to be considered in project scheduling are even more complicated, which makes developing a workable and suitable schedule is a more difficult task. Practically, the project schedule usually is developed based on scheduler's experiences. For example, the definition and the duration of activities, and the relationships between activities are determined by project planners. However, the result of project scheduling could be arbitrary since different project planners could have different prospective of developing the project networks.

To improve the aforementioned shortcomings, this research focuses on developing a scheduling model that can automatically generate a contract-driven schedule. Firstly, multi-dimensional (MD) attributes of building components are analyzed based on the standardized cost-code, which, by definition, is commonly used by the contractors to generate contract items. Then, those attributes are combined with 3D drawings to form MD objects. Hence, each MD object includes information about the required resources, such as labor, machinery, and material. Contractors can accurately calculate construction resources and estimate activity duration according to MD objects. Such an approach allows contractors to develop their own MD object database and generate new MD objects if new construction methods or new contract items are introduced.

Then, several principles are developed to obtain Object Relationship Matrix (ORM) and Object Similarity Matrix (OSM) based on the contractor's past experiences. These two matrixes contain the information about the construction method and approaches that the contractor would like to execute project. Genetic algorithms are then used with ORM and OSM to generate the optimal sequence of constructing building components. Finally, several constructive constraints, such as the resource limitation and the continuity of workflow, are also taken into consideration within the model development. A computer implementation called Project Planning and Scheduling System (PPSS) is developed to enhance the practice of the proposed model. In addition, a case study is presented to examine the efficiency of PPSS. It is proved that the automated scheduling model is capable to generate an optimal contract-driven schedule, and provides multi-dimensional information for management.

\section{PREVIOUS PROJECT SCHEDULING METHODS}

\subsection{The Shortcomings of Applying CPM for Scheduling}

Critical Path Method (CPM) has been widely applied for project planning. The project scheduler first analyzes the 
2D design drawings, then develop a Work Breakdown Structure (WBS) to list all required construction activities. The sequential relationships between activities, such as finish-start, finish-finish, start-finish, and start-start, are further defined. After evaluating the duration required for each activity, the project scheduler can prepare a project network accordingly to control construction progress. However, the relationships between activities are decided according to the experience and cognition of planners (Fischer and Aalami 1996). Consequently, the project schedule could be arbitrary could be arbitrary since different project planners could have different prospective of developing the project networks. In addition, Waly and Thabet (2002) point out, the relationships between building components, the supplement of resources, the situation of construction site, and the selection of construction method should be taken into consideration while developing the project schedule. However, construction project is usually divided to several subcontracts. These subcontracts are then awarded to several subcontractors. Therefore, projects are performed according to the contract-items. As a result, the actual activities at the construction site usually do not coincide with the pre-planned schedule. Due to the undefined relationships between activity and contract, activities are incapable to accurately reflect the real construction progress. As a result, the project schedule can only serve as a reference for progress management.

\subsection{Knowledge-Based Systems for Automated Scheduling}

To improve the shortcomings of CPM method, many researchers have taken the knowledge-based approach to schedule construction projects. Winstanley et al. (1991) propose OARPLAN (Object, Action, Resource, PLANer), and Stumpf et al. (1996) develop CPCA (Construction Project Control Agent). Both of them utilize the relationships between building components and scheduling rules to develop project schedules. Fisher and Aalami (1996) propose the CMM (Construction Method Model) that incorporates the impact factors, such as resource limitation and construction method, into scheduling. Accordingly, planners may apply what-if analysis to further analyze the results of applying different construction methods in terms of productivity, cost, duration, and etc. Also, Dzeng and Lee (2004) propose CasePlan, which is based on the selection of construction method and the relationships of objects, applies case-based reasoning and CPM for scheduling. And Waly and Thabet (2002) apply VCE (Virtual Construction Environment), which used virtual reality (VR) modeling techniques and object-oriented technologies, to simulate construction schedule.

\subsection{The Application of 4D Model}

Applying object-oriented technology to create object model for scheduling is another important research field. The object model not only contains geometric attributes, other attributes of resources and construction methods are also encapsulated. For example, combined 3D-Model with the scheduling, 4D Model which connected building components with activity items, and serves as a reference and simulation tool for scheduling (Stanford CIEF).

Several studies have shown the 4-D CAD model can be been successfully applied to project management. For example, Wang et al. (2004) apply the 4D-Model to establish the site management system. Dawood et al. (2004) propose an integrated database to simulate 4D/VR construction process. Although 4D-Model may present the time relationships between building components and some of their attributes, the description of other important attributes is insufficient to consider as a scheduling tool (Waly and Thabet 2002). Besides, since the 4D-Model is still design-driven, 4D CAD also can't flexibly react with the modification of project design or what-if analysis in the construction phase (Tanyer and Aouad 2005).

\section{MULTI-DIMENSIONAL MODEL}

\subsection{The Definition of MD Object}

The basic concept of MD Object is to systematically analyze building components in multiple disciplines, to generate its geometric, physical, behavioral and time-related attributes. Then, the object-oriented technology is applied to combine the analyzed attributes with CAD drawings. (Feng and Chen 2005).

To define the structure of the multi-dimensional attributes, a project is decomposed into several building components. Each component, by definition, is the smallest descriptive unit and presented in $3 \mathrm{D}$ form. In the geometric perspective, the attributes contain spatial information and physical relationships; in behavioral perspective, the contract items within building components are systematically analyzed to acquire resource information such as labor, machinery, and material. In time aspect, activity items are the combination of many contract items. The analyzed behavioral and geometric conditions are further used for scheduling. The structure of MD attributes is shown in Fig. 1.

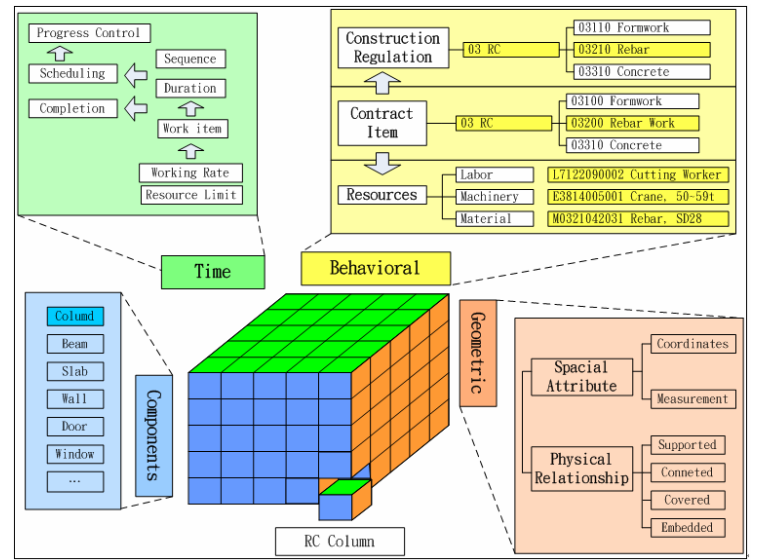

FIG. 1. The Structure of MD Attributes

3.2 The Application of MD Object for Project Scheduling 
As mentioned above, the CPM method uses activities for project scheduling. However, there is no full connection between activity and contract items. Therefore, to integrate information becomes labor-consuming and time-consuming. To solve the problem, this research uses MD Objects to connect activity with contract items, as shown in Fig. 2. Contract items of the building components and working rate are used to calculate each work item's duration. Then, the total duration of activities is obtained. Instead of directly using activity to calculate activity's duration, the proposed method may enhance the accuracy of duration's estimation. In the calculation of completion percentage, using the completed contract items of each component instead of the percentage of construction invoice may help managers accurately compare the actual progress with the estimated progress. In scheduling, when any modification occurs or rescheduling is necessary to reflect actual construction situation, the MD Objects may promptly and flexibly provide managers an optimal solution.

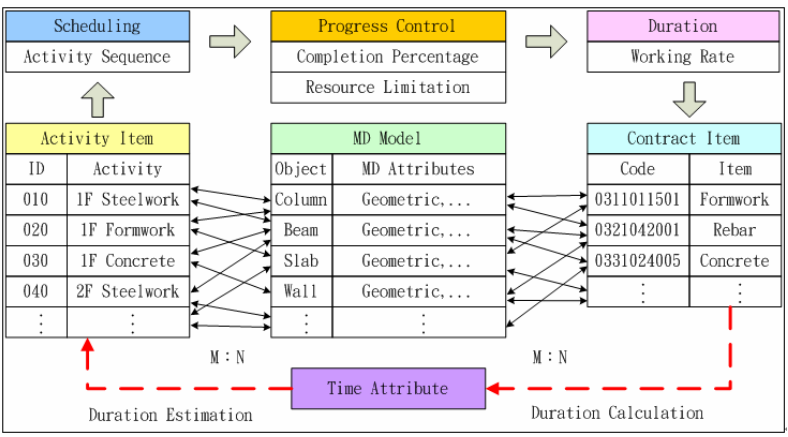

FIG. 2. The Analysis of Time Attribute

\subsection{The Creation of MD Model}

This research applies the Architectural Desktop 2006 (ADT2006), which is developed by AutoDesk Company, for the creation of MD Model. As noted, same components have been repeatedly applied in a construction project. To facilitate the process of creating project's MD Model, the default MD Objects such as beam, column, wall and slab are systematically saved in a MD Object Database (MDODB). In the design phase, users may select MD Objects from MDODB and input parameters, according to any specific requirements. The parameter-input interface of column component is shown in Fig. 3. After the process of selecting MD Objects and inputting parameters, a MD Model is created (as shown in Fig. 4) and presented in 3D form, and the multiple construction information of MD Objects is integrated to a database for information management and integration purpose.

\section{THE PROPOSED SCHEDULING MODEL}

\subsection{The Impact Factors of Scheduling}

In fact, it is hard to consider all factors of scheduling. However, planners may select appropriate impact factors, according to their previous experiences and the characteristics of the project. In the development of automated scheduling model, this paper will consider two impact factors, which are the physical component relationship and trade interaction, respectively. There are four relationships discussed in the physical component relationship, which are "supported by", "connected to", "covered by", and "embedded in". And there are also three restrictions in trade interaction, which are zoning, resource limitation and workflow.

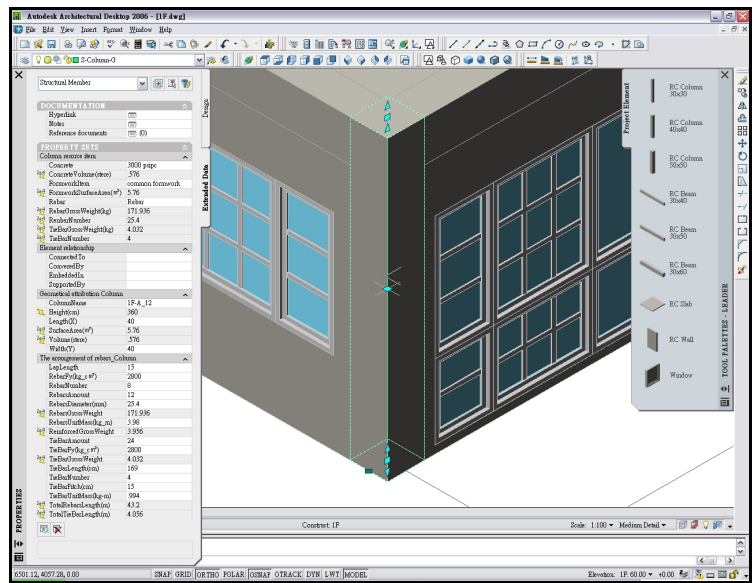

FIG. 3. Dialog Box for Entering Column's Parameters
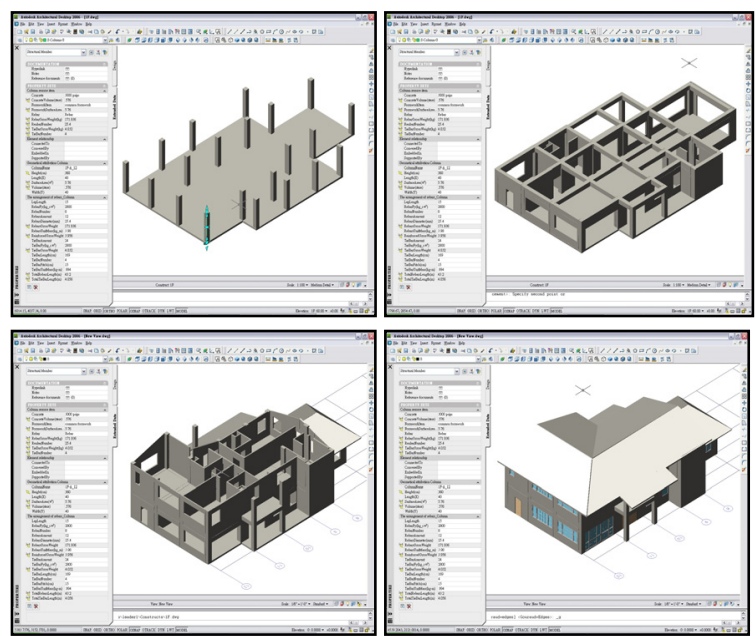

FIG. 4. The Creating Process of Project's MD Model

\subsection{Object Relationship Matrix}

The physical relationship between building components is presented in a matrix-called the Object Relationship Matrix (ORM), In a ORM, vertical axis refers to active components and horizontal axis refers to passive components. For example, in Fig. 5, the circled $\mathrm{C}$ in the left means object L (wall) is "connected to" object A (column), and the circled $S$ in the right means object $G$ (beam) is "supported by" object I (wall). This research only involves discussion on the direct relationship between components (i.e. joint components), excluding the indirect relationship between components. The sequence of objects in ORM is the sequence of constructing building components. As shown in Fig. 5, object $\mathrm{G}$ is supported by object I, which means that object I must appear before object $\mathrm{G}$, therefore, object I should be moved to the front of object $\mathrm{G}$. That is, 
the object relationship in the upper-right zone of the matrix should all be moved to the lower-left zone, which is the rational sequence of objects.
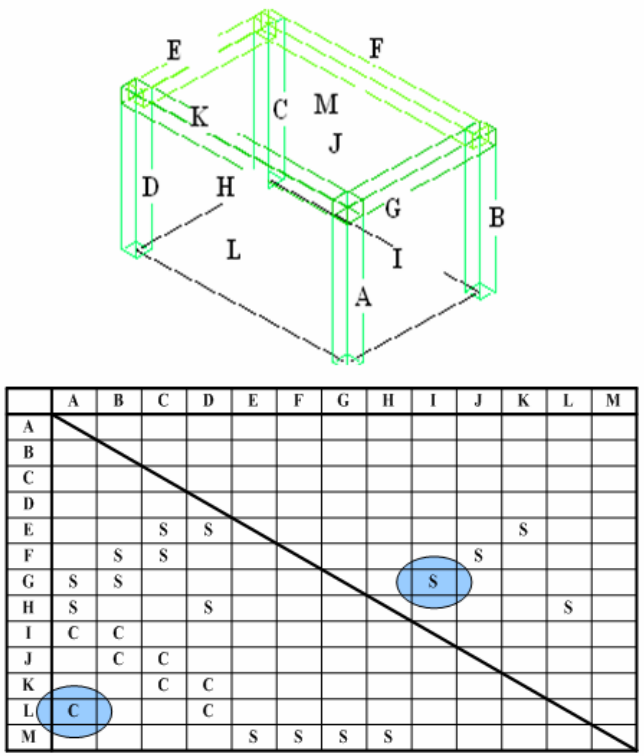

FIG. 5. Object Relationship Matrix

\subsection{Object Similarity Matrix}

Besides ORM, the Object Similarity Matrix (OSM) should be defined. The ORM matrix presents the rationality of objects' sequence, but the OSM matrix provides information on the continuity of construction. In Fig. 6, S represents similarity, and the circled $\mathrm{S}$ indicates that object $\mathrm{H}$ and object $\mathrm{E}$ are similar (both are columns).

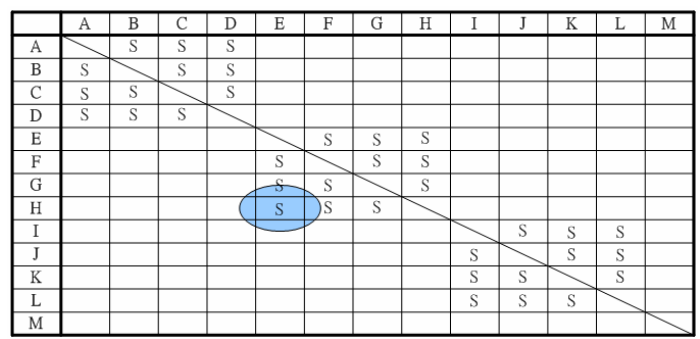

FIG. 6. Object Similarity Matrix

\subsection{The Application of GA}

Genetic Algorithms (GA) are search algorithms developed by Holland (1975), which are based on the mechanics of natural selection and genetics to search through decision space for optimal solutions. The metaphor underlying GA is natural selection. In evolution, the problem each species faces is to search for beneficial adaptations to the complicated and changing environment. In other words, each species has to change its chromosome combination to survive in the living world. In GA, a string represents a set of decisions (chromosome combination), a potential solution to a problem. Each string is evaluated on its performance with respect to the fitness function (objective function). The ones with better performance (fitness value) are more likely to survive than the ones with worse performance. Then the genetic information is exchanged between strings by crossover and perturbed by mutation. The result is a new generation with (usually) better survival abilities. This process is repeated until the strings in the new generation are identical, or certain termination conditions are met. Fig. 7 demonstrates the general structure of Genetic Algorithms. Goldberg (1989) and Gen and Cheng (1999) are good references for details regarding Genetic Algorithms.

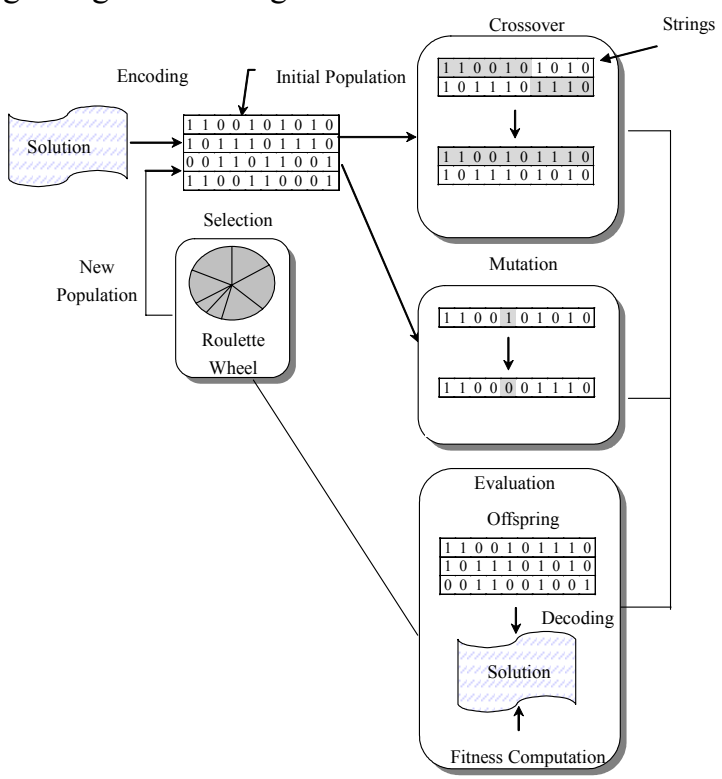

FIG. 7. General Structure of Genetic Algorithms (adopted from Gen and Cheng, 1999)

In this paper, the chromosome strings are encoding in real number. Each code represents a single object, as shown in the following Fig. 8. The initial genetic strings are input into $x(i, j)$, while $i$ is the size of initial population (1 Pop_Size), and $j$ is the number of objects $(j=1 \sim$ total number of objects).

\begin{tabular}{|c|c|c|c|c|c|c|c|c|c|c|c|c|c|}
\hline Genetic Code & 2 & 13 & 12 & 1 & 11 & 6 & 5 & 4 & 9 & 3 & 10 & 7 & 8 \\
\hline Object ID & B & M & $\mathrm{L}$ & A & K & $\mathrm{F}$ & E & D & I & C & $\mathrm{J}$ & G & H \\
\hline
\end{tabular}

FIG. 8. The Chromosome Structure

Before creating a fitness function, the appropriate weights of ORM and OSM should be defined. The setting of weight is illustrated as followed. In ORM, the weight between the objects with the hard constraint from the above example defined as 5 , other objects without hard constraints are defined as 0, as shown in Fig. 9.

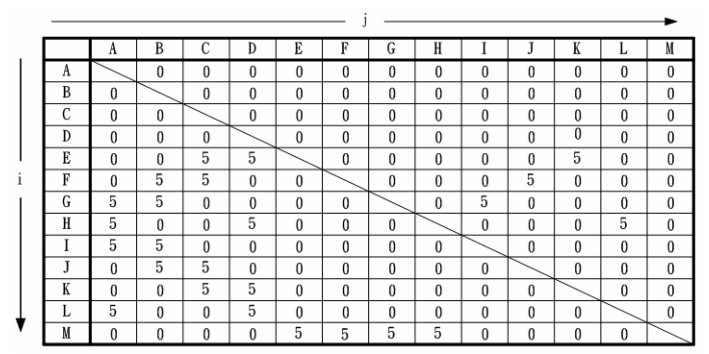

FIG. 9. The Appropriate Weight in ORM 
As mentioned above, the rational sequence of objects is to move the object relationship in the upper-right zone of the matrix to the lower-left, i.e. to minimize the weight value of the upper-right zone. Hence, the fitness function in this model is given as Eq. (1) :

$$
\text { Min. } f(x)=\left(\sum_{i=1}^{12} \sum_{j=i+1}^{13} x(i, j)\right)
$$

Modify the fitness function to be in maximization form: Eq. (2).

$$
\text { Min. } f(x)=\text { Max. } g(x)=\operatorname{Max} .\{-f(x)\}
$$

Applying the above function, the fitness function is transferred into Eq. (3) :

$$
\text { Max. Fitness Value }=-\left(\sum_{i=1}^{12} \sum_{j=i+1}^{13} x(i, j)\right)+C
$$

Among which, the constant value $\mathrm{C}$ is decided by the sum of weight value in ORM. For example, the sum of weight value in Fig. 9 reads 120, then the constant value $C$ can be set as 0 , to show that the formula's convergence condition while the weight of the upper-right is 0 .

The result obtained through ORM and GA is only a rational sequence of objects, the continuity of construction should be also taken into consideration. Hence, according to the similarity of objects, a penalty value should be given to the fitness function. Therefore, only considering both the rationality of objects' occurrence and the continuity of construction, the obtained sequence of objects will be conform to the actual situation of construction. In OSM, the weight set by ORM is referred to decide the weight of OSM. If the ORM is hard constraint, the rational sequence of objects is more important than the continuity of construction, therefore, a penalty value of 0.01 is given to non-similar objects, as shown in Fig. 10.

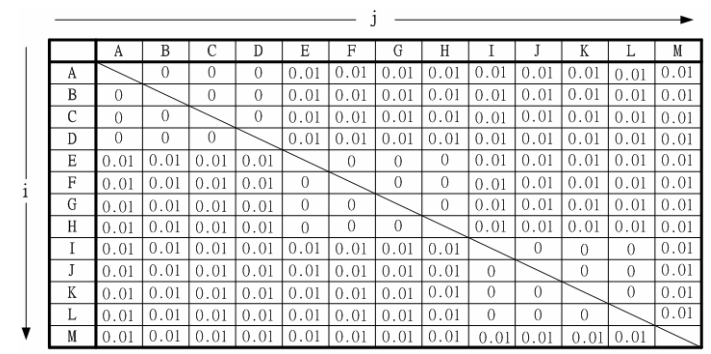

FIG. 10. The Appropriate Weight of OSM

Then, the fitness function is revised as Eq. (4).

Max. Fitness Value $=-\left(\sum_{i=1}^{12} \sum_{j=i+1}^{13} x(i, j)+y(i, j)\right)+C$

$y(i, j)$ is the penalty function. And the constant value $\mathrm{C}$ : $C=\operatorname{Sum}($ Weight - ORM $)+\operatorname{Sum}($ Weight - OSM $)$

After GA's evolution and convergence procedure, the obtained sequence of objects has considered both the physical relationship of objects and the continuity of construction. Therefore, this sequence can be further served as a basis on scheduling.

\section{A CASE STUDY ON AUTOMATED SCHEDULING}

\subsection{Project Planning and Scheduling System}

To integrate information based on MD Model, the proposed Project Planning and Scheduling System (PPSS) is developed. There are 4 sub-systems in the PPSS system: "Project Information', "Object Physical Constraints", "Scheduling Process", and "Progress Control", as shown in Fig. 11. In "Project Information", all project information is recorded. It also provides inquiry functions to search for the information related to the contract items, the quantity of required resources, etc. In Object Physical Constraints, the physical relationship and similarity information of building components are provided to create the ORM and OSM matrices. Information, such as the parameters of GA, the start-finish dates of a project, and the working rate, are given in the "Scheduling Process" sub-system. As for "Progress Control", there are three main functions: to establish bar chart, to record completed item, and to control progress and rescheduling.

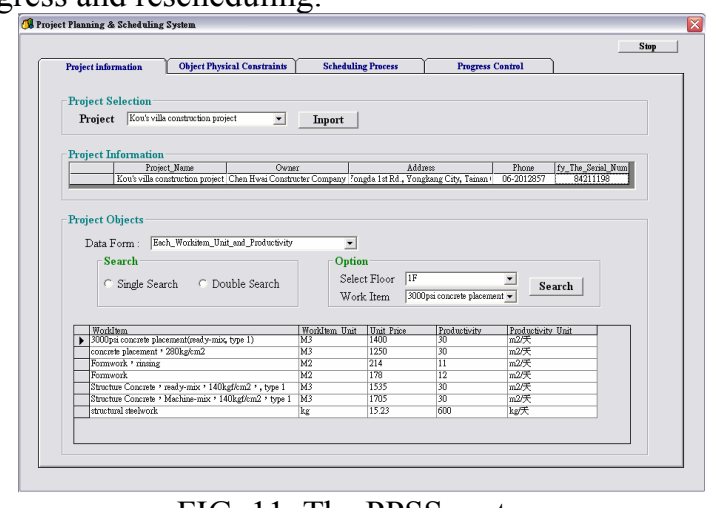

FIG. 11. The PPSS system

\subsection{Case Study}

The structural engineering of a private villa's construction project is studied to evaluate the PPSS system. Firstly, a MD Model is created according to construction drawings and contract documents. The information on multi-dimensional attributes is output to a project database and further used for information management and progress control in the PPSS system. In terms of information integration, using traditional method to handle construction data might lead to calculation-error and be time consuming. Compared with the manual quantitative estimation, the estimated outcome from PPSS system is more accurate, and any change of information in MD model will be simultaneously updated. Therefore, it is proved that PPSS may provide accurate and prompt information for the use of scheduling and progress management.

In scheduling, according to the object relationship of MD Model, the Object Physical Constraints is created, and the ORM and OSM matrices are generated according to the selected area (as shown in Fig. 12). In Scheduling Process section, the parameters of GA (number of population, mutation rate, crossover rate, numbers of generations), the start-finish date of the project, working rate, etc., are defined, and through GA, the optimal sequence of constructing building components and contract items are 
decided. As for Progress Control, project is scheduled according to objects' physical constraint, work flow path, resource limitation, and other impact factors (as shown in Fig. 14). In the implementation of scheduling, a project is zoned for scheduling, and each zone schedule is then integrated to present a whole project schedule. In this way, the discrepancy of scheduling outcome can be avoided. In construction phase, the completed building component is recorded in the "Finish" column. The percentage of completed construction works is calculated according to the completed contract items, to accurately reflect the construction progress. If actual progress doesn't follow the project planning, the working rate should be revised and the incomplete items will be rescheduled to match the project's objective in terms of time and cost.

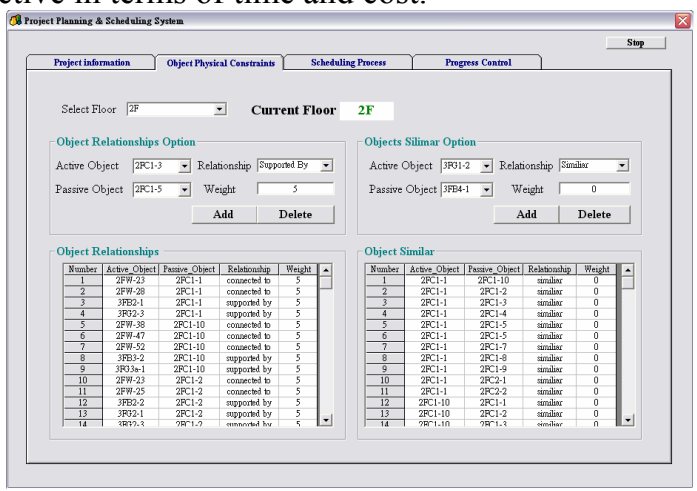

FIG. 12. Object Physical Constraints

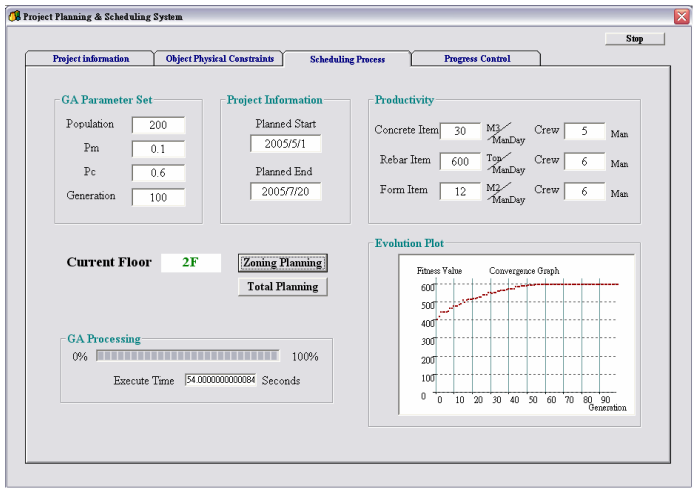

FIG. 13. Scheduling Process

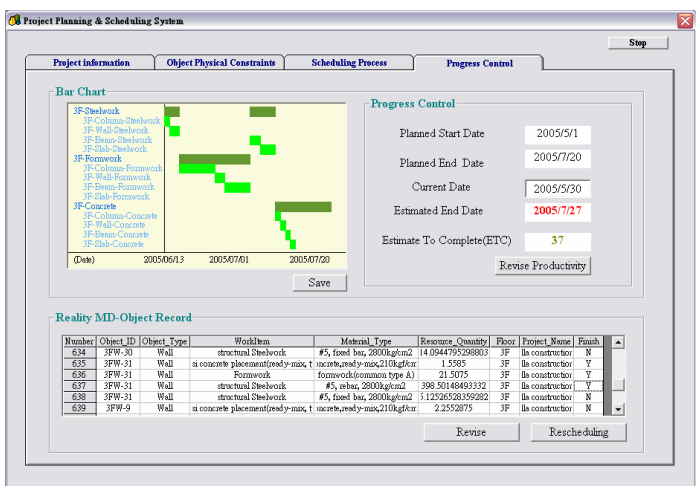

FIG. 14. Progress Control

\section{CONCLUSION}

Based on MD CAD Objects and MD model, this paper provides a new foundation for the development of automated scheduling system. Efficient as an integration platform of project information, the MD model ensures the accuracy and instantaneousness of information transfer and exchange. In terms of the flexibility of project planning, with the help of the proposed contract-driven scheduling system, project schedulers may perform what-if analysis and re-scheduling according to the actual construction condition. As to the efficiency of progress control, with the help of the PPSS system, the construction situation is precisely recorded. Hence, by comparing the actual progress with the scheduled, any delay in progress will be noticed and handled.

\section{REFERENCES}

[1]Dawood,N., Akinsola,A., Hobbs,B., "Development of automated communication of system for managing site information using internet technology", Automation in Construction, 11 (2002) 557-572.

[2]Fischer, M. A., and Aalami, F., "Scheduling with computer-interpretable construction method models", ASCE J. Constr. Eng. Manag., 122 (4) (1996) 337-347.

[3]Waly, A. F., and Thabet, W. Y., "A virtual construction environment for preconstruction planning", Automation in Construction, 12 (2002) 139-154.

[4]Wanstanley, G., Chacon, M. A., Levitt, R. E., “An integrated project planning environment", Intelligent. System Engineering, 2 (2) (1991) 91-106.

[5]Stumpf, A. L., Ganeshan, R., Chin, S., Liu, L. Y., "Object-Oriented model for integrating construction product and process information", ASCE J. Comput. Civ. Eng., 10 (3) (1996) 204-212.

[6]Dzeng, R. J., and Lee, H. Y., 'Critiquing contractors' scheduling by integrating rule-based and case-based reasoning", Automation in Construction, 1 (2004) 665-678.

[7]CIFE web site, http://www.stanford.edu/group/4D/index.shtml

[8]Wang, H. J., Zhang, J. P., Chau, K.W., Anson, M., “4D dynamic management for construction planning and resource utilization", Automation in Construction, 13 (2004) 575-589.

[9]Dawood, N., Sriprasert, E., Mallasi, Z., Hobbs, B., "Development of an integrated information resource base for 4D/VR construction processes simulation", Automation in Construction, 12 (2002) 123-131.

[10]Tanyer, A. M., and Aouad, G., "Moving beyond the fourth dimension with an IFC-based single project database", Automation in Construction, 14 (2005) 15-32.

[11]Feng, C. W., Chen, Y. J., "Using MD CAD Objects to Integrate Information for Construction Projects", Proceedings of ICCEM, Korea, pp.515 519 (2005).

[12]Holland, J., Adaptation in Natural and Artificial System, University of Michigan Press, Ann Arbor, Mi., 1975.

[13] Goldberg, D. E., Genetic algorithms in search, optimization and machine learning, Addition-Wesley, Reading, MA, 1989.

[14]Gen, M., and Cheng, R., Genetic Algorithms and Engineering Optimization. Wiley, New York, 1999. 